\title{
Towards assembly completion and preparation of experimental campaigns of Wendelstein 7-X in the perspective of a path to a stellarator fusion power plant
}

\author{
T. Klinger, C. Baylard, C. D. Beidler, J. Boscary, H.S. Bosch, A. Dinklage, D. Hartmann, \\ P. Helander, H. Maßberg, A. Peacock, T. S. Pedersen, T. Rummel, F. Schauer, L. Wegener, R. Wolf \\ Max-Planck Institute for Plasma Physics, Greifswald, Germany
}

\begin{abstract}
The superconducting stellarator device Wendelstein 7-X, currently under construction, is the key device for the proof of stellarator optimization principles. To establish the optimized stellarator as a serious candidate for a fusion reactor, reactor-relevant dimensionless plasma parameters must be achieved in fully integrated steady-state scenarios. After more than 10 years of construction time, the completion of the device is now approaching rapidly (mid 2014). We discuss the most important lessons learned during the device assembly and first experiences with coming major work packages. Those are (a) assembly of about 2500 large, water-cooled, 3d-shaped in-vessel component elements; (b) assembly of in total 14 superconducting current leads, one pair for each coil type, (c) assembly of the device periphery including diagnostics and heating systems. In the second part we report on the present status of planning for the first operation phase $(5-10 \mathrm{~s}$ discharge duration at $8 \mathrm{MW}$ heating power), the completion and hardening of the device for full power steady-state operation, and the second operation phase (up to $30 \mathrm{~min}$ discharge duration at $10 \mathrm{MW}$ heating power). It is the ultimate goal of operation phase one to develop credible and robust discharge scenarios for the high-power steady-state operation phase two. Beyond the improved equilibrium, confinement, and stability properties owing to stellarator optimization, this requires density control, impurity control, edge iota control as well as high density microwave heating. Of paramount importance is the operation of the island divertor, which is realized in the first operation phase as an inertially cooled conventional graphite target divertor. It will be replaced later on by the steady-state capable island divertor with its water-cooled carbon fiber reinforced carbon target elements.
\end{abstract}

Keywords: stellarator, superconductivity, lessons learned, optimization, steady state operation, fusion power plant

\section{The device}

The stellarator Wendelstein $7-\mathrm{X}$ is a superconducting fusion device with optimized magnetic field geometry and a modular coil concept. After completion it will be the largest optimized stellarator in the world with a plasma volume of $30 \mathrm{~m}^{3}$. It is designed to operate a reactor-relevant plasma under steady-state conditions (up to $1800 \mathrm{~s}$ discharge duration, cf. also Sec. 3). The magnetic field configuration of Wendelstein 7-X is the result of a dedicated optimization process based on a suite of numerical codes [1]. The periodicity of the magnetic field is five-fold and consequently the device comprises five nearly identical magnet modules. A schematic drawing of Wendelstein 7-X is shown in Fig. 1. The most important parameters of the device are compiled in Tab. 1.

Each module consists of two flip-symmetric half modules with five different non-planar and two different planar coils each. Each of the seven coil types are independently energized to provide a wide range of operational flexibility, in particular the rotational transform and the mirror ratio. In addition there are two control coils and one trim coil per module for further experimental flexibility and compensation purposes. The 50 non-planar and 20 planar 


\begin{tabular}{ll} 
physics parameter & parameter value \\
\hline \hline plasma volume & $V_{p}=30 \mathrm{~m}^{3}$ \\
magnetic induction on axis & $B_{0}=2.5-3 \mathrm{~T}$ \\
magnetic field energy & $E_{\text {mag }}=600 \mathrm{MJ}$ \\
field periodicity & 5 \\
rotational transform & $\nmid=5 / 6 \ldots 5 / 4$ \\
ECRH heating power & $P_{E C R}=10 \mathrm{MW}(1800 \mathrm{~s})$ \\
NBI heating power & $P_{N B I}^{D+}=10 \mathrm{MW}(10 \mathrm{~s})$ \\
ICRH heating power & $P_{I C R}=5 \mathrm{MW}(10 \mathrm{~s})$ \\
pulse length & $t_{p}=1800 \mathrm{~s}(\mathrm{at} 10 \mathrm{MW})$ \\
energy turnover & $E_{t o}=18 \mathrm{GJ}$ \\
\hline \hline & \\
engineering parameter & parameter value \\
\hline \hline major radius & $5.5 \mathrm{~m}$ \\
minor radius & $0.53 \mathrm{~m}$ \\
number of non-planar coils & 50 \\
number of planar coils & 20 \\
number of current leads & 14 \\
number of ports & $254 \mathrm{of} 120$ types \\
machine height & $4.5 \mathrm{~m}$ \\
outer diameter & $16 \mathrm{~m}$ \\
total mass & $750 \mathrm{t}$ \\
total cold mass & $425 \mathrm{t}$ \\
\hline \hline
\end{tabular}

Table 1: Key physics and engineering parameters of the stellarator Wendelstein 7-X. The listed values for the plasma heating powers refer to the second operation phase (cf. Section 3); the initial values are $P_{E C R}=8 \mathrm{MW}$ and $P_{N B I}^{H+}=3.5 \mathrm{MW}$, respectively (no ICRH foreseen).

coils are superconducting (liquid He cooled NbTi cablein-conduit with $18.2 \mathrm{kA}$ and $16 \mathrm{kA}$ nominal current, respectively) and are designed to produce a magnetic induction of up to $3 \mathrm{~T}$ on the magnetic axis. To react to the magnetic forces, planar and non-planar coils are fastened to a cast steel central support structure by a bolted connection with possible gap opening at the flanges. Dedicated support elements connect mechanically adjacent coil casings on both the high- and the low-field side of the machine. The so-called narrow support elements are sliding contacts, while the lateral support elements are welded connections with the exception of the (bolted) inter-module support elements. The support concept is a compromise between stiffness and flexibility of the magnet mechanical system in order to minimize the relative movement of the coils while keeping the maximum stress values within the allowables [2]. The cold mass (coils and support system) is $425 \mathrm{t}$ in total and is cooled with liquid He to $3.4 \mathrm{~K}$. The cold components are in the cryostat volume which is formed by the plasma vessel, the outer vessel and the ports. The 254 ports are designed and optimized for plasma diagnostics, sensors, heating, water supply of in-vessel components, and vacuum pump-down. The ports are welded on corresponding openings in the plasma vessel and domes on the outer vessel. They are equipped with bellows to compensate for deformations and displacements between plasma vessel and outer vessel during pump-down and wall heating. All vessel and port outer surfaces are insulated by a multilayer insulation (Al-coated Kapton ${ }^{\circledR}$ foil) which is covered with a thermal shield (glass fibre composite on the plasma vessel and brass on the outer vessel surface with He gas cooling to $70 \mathrm{~K}$ ) to minimize the heat load on the cryogenic components. The in-vessel components comprise the divertor targets and baffles, vertical and horizontal closures, wall panels and heat shields for wall protection. For each of the ten divertor modules, a control coil is foreseen for strike-point position control; a cryo-pump system will be installed in each of the divertor modules later for steady state operation. All in-vessel components are pressure water-cooled. A complex cooling pipe system, sensors, cables, and a number of embedded diagnostics complete the in-vessel system.

\section{Assembly status and lessons learned}

For about five years the project Wendelstein 7-X has been (and remains) on track, i.e., on budget and on schedule. All major device components are manufactured, tested and delivered. Large assembly work packages with considerable project risks have been successfully completed or are close to completion (assembly of the vessels, magnet system, support elements, bus bar system, He pipe system, thermal insulation, ports $[5,6])$. A photo of the present view into the torus hall is shown in Fig. 2. The remaining large assembly work packages are the following:

1. Wall elements, water pipes and graphite divertor modules inside the plasma vessel (2500 main components, $265 \mathrm{~m}^{2}$ total surface, $34 \mathrm{t}$ total weight, see Fig. 3), 


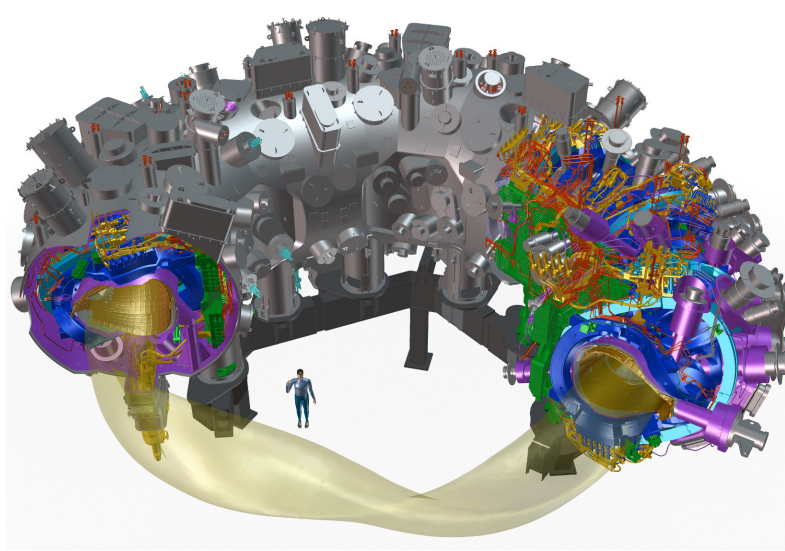

Figure 1: Schematic diagram of the superconducting stellarator Wendelstein 7-X. Shown are non-planar coils (dark blue), planar coils (light blue), He pipes (red), superconducting bus bar system and current leads (yellow), thermal insulation (magenta), in-vessel components (gold). Also shown are the cryostat vessel (light grey) and the machine base (dark grey).

2. high temperature $18.2 \mathrm{kA}$ superconducting current leads ( 7 go and 7 return bars $=14$ single elements),

3. device periphery, i.e., steel support structures, platforms, water and He pipework, device instrumentation, cable trays, diagnostics, heating systems.

The completion of the device is still foreseen for mid2014 and the detailed planning for the device commissioning is in progress. The above listed work packages are challenging because of (1) three-dimensional geometry and high precision requirements, (2) difficult access to the cryostat, (3) the extremely crowded space situation in the torus hall. Generally this leads to high work density and sensitivity against perturbations in the work flow. Intense project management on the daily level is required, based on strict rules and well defined processes, in particular systematic quality management, change management and a pragmatic risk management.

The project Wendelstein 7-X was troubled for many years by delays in component deliveries, quality deviations in major components, a significant increase in design and assembly effort, lack of engineering capacity, and, last but not least, inadequate managerial processes and a lack of project-oriented work style. The recovery of the project took years and required a substantial effort by all involved parties. As a matter of fact, big science

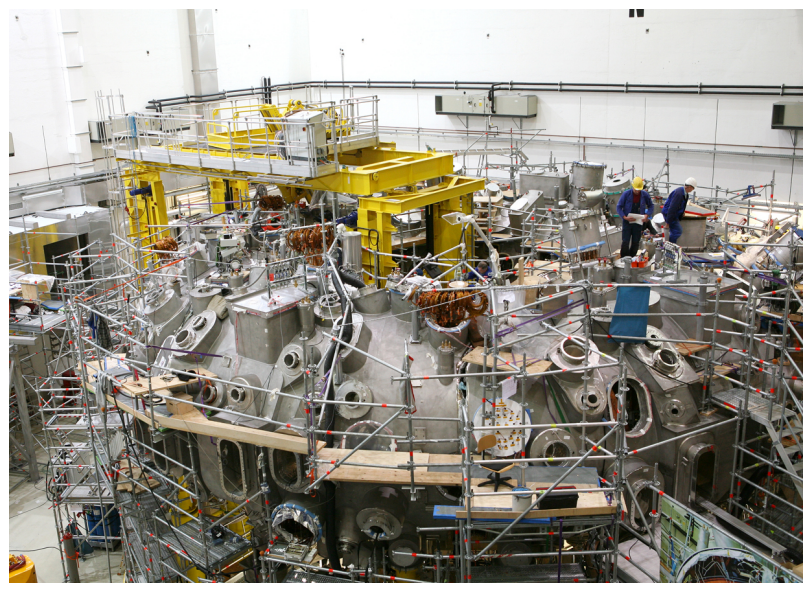

Figure 2: View into the experiment hall. The torus is now closed and just the outer vessel with its openings and domes is visible. The port assembly bridge (yellow) is in use for the few remaining ports to be installed. Also seen is the extensive scaffolding necessary for the access to the device.

projects always bear a significant risk which is very much determined by the projects' specific boundary conditions. Nevertheless, there are a few - admittedly rather general lessons learned that should be taken seriously into account to mitigate the project risks as much as ever possible.

Lesson 1:. A lack of generous margins, clearances and reasonable tolerance levels implies an unnecessary increase of the complexity and leads to late design changes. This has a strong impact on schedule, budget, man-power and potentially sours the relationship to funding bodies.

Lesson 2: Major components should be subject to a rigorous qualification program prior to tender action. The actual manufacturing must be accompanied by a dedicated test program. Qualified inspectors and quality management teams must follow up manufacturing processes in a very detailed way.

Lesson 3:- Development and manufacturing risks must be taken to a large extent by the project. Industry cannot accept high risks or will charge the project to cover unexpected costs, even beyond the contract. The only way is to solve all problems step-by-step together with the industrial partner. 


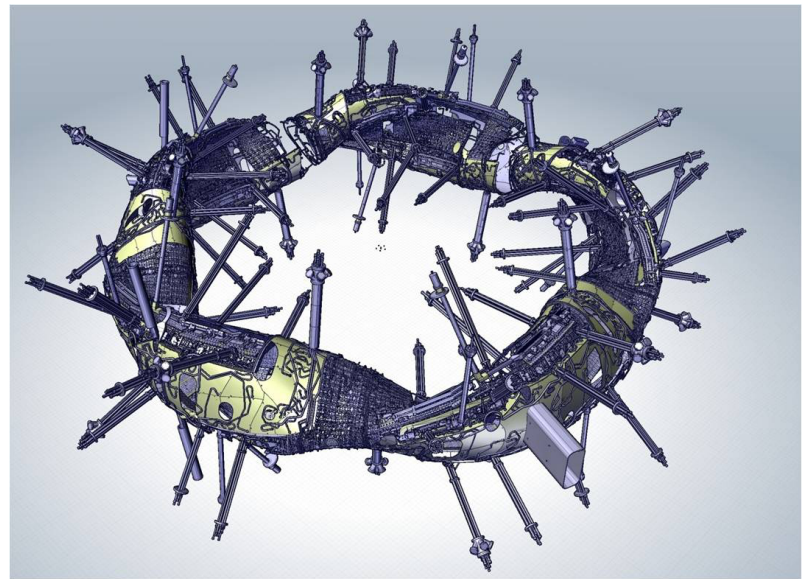

Figure 3: Model of the complete in-vessel system including watercooled steel panels, water-cooled graphite heat shields, divertor modules with baffle and target plates, water pipes and port plug-ins.

All three lessons are directly derived from experiences in the project Wendelstein 7-X. Indeed, much of the apparent complexity of the device is not specific to stellarators and their three-dimensional geometry but is the consequence of the lack of margins and clearances, late design changes and technical solutions that go far beyond the industrial standards. The next step device on the optimized stellarator line should be very much simplified, based on a systematic engineering optimization that must comply with the goals of the physics optimization of the stellarator magnetic field (cf. Sec. 4).

\section{Physics program}

Stellarators are inherently steady-state capable since in contrast to tokamaks - a net toroidal current flowing in the plasma is not required. The rotational transform of the magnetic field is provided entirely by the currents flowing in external coils and its profile may be chosen to avoid low-order resonances and their accompanying islands in the confinement volume and instead to place such islands at the plasma boundary where their $x$-points provide a naturally occurring 'island divertor' [7]. However, the classical stellarator needs physics optimization $[1,3,4]$ to allow for integrated discharge scenarios with reactor-relevant plasma parameters. These integrated scenarios require simultaneously (a) true steady-state oper- ation with discharges of several minutes to ensure that equilibrium is reached with plasma facing components, (b) high densities and temperatures, (c) full density control with continuous pellet refueling, (d) viable divertor performance with high radiated-power fraction and detachment, (e) no impurity accumulation in the core. We underline that it is extremely challenging - though possible - to meet all these requirements at the same time and a dedicated, well-focused research plan for Wendelstein $7-\mathrm{X}$ is needed. Such a plan should be able to address the most important research needs:

1. The efficacy of stellarator optimization must be proven. The physics of optimization is well understood and most points can be adequately investigated already during the first few years of operation. Even with limited heating power, first elements of high$<\beta>$ plasmas and fast particle confinement (isodynamic drift optimization) can be studied.

2. Discharges with simultaneously high densities, high temperatures, and good energy confinement times must be achieved. The energy confinement times must be demonstrated to be as good as for a similar size tokamaks $\left(\tau_{E}=0.1-1 \mathrm{~s}\right)$, in relevant discharge regimes with low collisionality, high $-\langle\beta\rangle$ and high- $n T \tau$.

3. Density (profile) control must be achieved to produce flat profiles at high densities $\left(n_{e} \geq 10^{20} \mathrm{~m}^{-3}\right)$. This most likely requires continuous central fuelling with pellets since the neoclassical thermodiffusion in stellarators may lead to hollow density profiles [8].

4. High-density operation without impurity accumulation must be demonstrated. This is a critical issue since at high densities with $T_{i} \approx T_{e}$ the ambipolar electric field tends to be negative in a stellarator implying rapid impurity accumulation thus leading to radiation collapse in the ELM-free H-mode. The high-density H-mode (HDH mode) [9] might well be a solution, combining high energy confinement time with low impurity confinement time. Both effects were experimentally observed in the predecessor device Wendelstein 7-AS [10]. The physics of the HDH-mode is, however, poorly understood and a similar behavior is to be demonstrated in Wendelstein 7-X.

5. Viable divertor performance is to be demonstrated 
with full density control, partial detachment, and $90 \%$ radiated power fraction at high density $n_{e} \leq$ $2 \cdot 10^{20} \mathrm{~m}^{-3}$ at steady-state $10 \mathrm{MW} / \mathrm{m}^{2}$ heat load.

6. Microwave heating of high density plasmas must be demonstrated since $f=140 \mathrm{GHz}$ ECR is the only steady-state heating scheme on Wendelstein 7-X. Above the X2-cutoff density $n_{\text {co X2 }}=1.2 \cdot 10^{20} \mathrm{~m}^{-3}$ at $B_{0}=2.5 \mathrm{~T}$ one needs multi-pass O2-heating. Overdense plasmas with $n_{e} \gtrsim 2 \cdot 10^{20} \mathrm{~m}^{-3}$ require schemes based on OXB-mode conversion [11]. For the control of the edge rotational transform, a modest ECR current drive might be used [12].

From this list of research needs a first approach towards a research plan can be derived. The actual planning work will be done jointly with the international partners that will become a key element in the scientific exploitation of Wendelstein 7-X. There is a sound knowledge base on modern stellarator physics, grounded on results from smaller devices (e.g. Ref. [13]) as well as the Large Helical Device (LHD, presently the largest stellarator in the world) [14] and the Wendelstein 7-X predecessor device Wendelstein 7-AS [10]. Fig. 4 shows a first concept for the research program of the first operation phase, where the heating power is limited $\left(P_{E C R}=8 \mathrm{MW}\right.$ and $\left.P_{N B I}^{H+}=3.5 \mathrm{MW}\right)$ and the divertor modules are based on inertially cooled graphite tiles. This 'test divertor' has the same geometry as the high-heat-flux divertor but is more robust against overloads. However, the discharge duration at full power is then limited to $5-10 \mathrm{~s}$. The major goal of

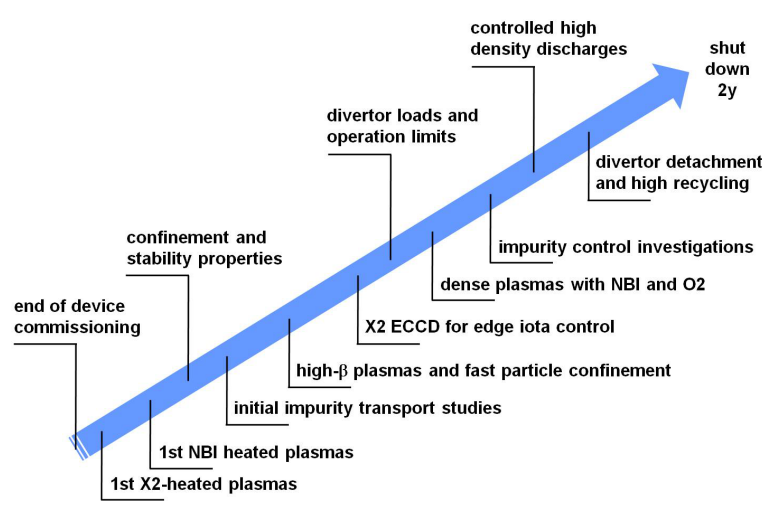

Figure 4: Sequence of investigations to be performed during the first operation phase of Wendelstein $7-\mathrm{X}$. the first operation phase is to develop credible and stable discharge scenarios for steady-state high-power divertor operation and to explore related operation windows.

For the second operation phase more heating power will be available $\left(P_{E C R}=10 \mathrm{MW}, P_{I C R}^{H+}=5 \mathrm{MW}, P_{N B I}^{H+}=\right.$ $\left.7 \mathrm{MW}, P_{N B I}^{D+}=10 \mathrm{MW}\right)$ and the inertially cooled divertor is replaced (during a shut-down of two years) by the steady-state capable high-heat-flux $\left(P_{\text {target }}=10 \mathrm{MW} / \mathrm{m}^{2}\right)$ divertor with actively cooled carbon fiber composite target elements. This opens the gate towards true highpower steady-state operation and the related steps shown in Fig. 5 go systematically in that direction. It is the ultimate goal of the second operation phase to combine high plasma performance parameters with long, well controlled discharge times.

\section{Stellarator reactor}

Even if all topics listed in the previous section can be addressed, Wendelstein $7-\mathrm{X}$ will not be able to tackle all the relevant issues in the physics of burning plasmas. Much of the technology development and also operation experience from ITER will be valuable for the development of a stellarator FPP, but there are also specific differences between the physics of tokamaks and stellarators. One is the combined effect of neoclassical and turbulent transport, another example is the much richer spectrum of unstable Alfvén eigenmodes driven by fast particles [15], the third one is the physics of the island divertor

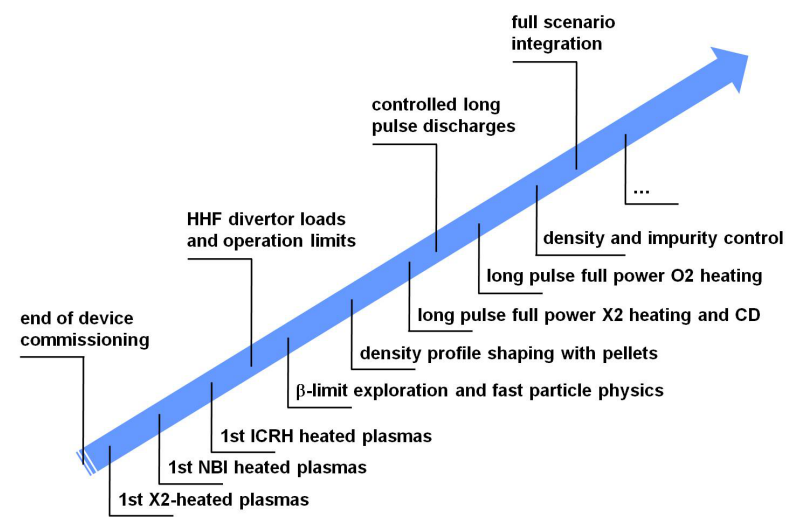

Figure 5: Sequence of investigations to be performed during the second operation phase of Wendelstein 7-X. 
[7]. A direct extrapolation of Wendelstein 7-X to a stellarator fusion power plant (FPP) would therefore not be without risk of failure, although the degree of risk would certainly be reduced by significant progress in the theoretical models used to explain (and subsequently predict) Wendelstein 7-X and ITER results. Given current knowledge, a more prudent approach would require a stellarator ignition experiment as an intermediate step. Tab. 2 shows key parameters of a fusion power plant study [16], largely based on the Wendelstein 7-X design but with numerous improvements and simplifications.

\begin{tabular}{lll} 
quantity & Wendelstein 7-X & stellarator FPP \\
\hline \hline$B_{0}$ & $2.5-3 \mathrm{~T}$ & $5-6 \mathrm{~T}$ \\
$V_{p}$ & $30 \mathrm{~m}^{3}$ & $1500 \mathrm{~m}^{3}$ \\
$P_{h}$ & $20-30 \mathrm{MW}$ & $0.6 \mathrm{GW}(\alpha) 3 \mathrm{GW}($ fus $)$ \\
$\mathrm{ECR}$ & $140 \mathrm{GHz}(\mathrm{X} 2, \mathrm{O} 2)$ & $140 \mathrm{GHz}(\mathrm{O} 1)$ \\
$<\Gamma_{n}>$ & negligible & $1 \mathrm{MW} / \mathrm{m}^{2}$ \\
$<\Gamma_{h}>$ & $0.1 \mathrm{MW} / \mathrm{m}^{2}$ & $0.4 \mathrm{MW} / \mathrm{m}^{2}$ \\
\hline \hline
\end{tabular}

Table 2: Key physics and engineering parameters of a stellarator fusion power plant in comparison with Wendelstein 7-X. $B_{0}$ toroidal magnetic induction on axis, $V_{p}$ plasma volume, $P_{h}$ heating power, $\mathrm{ECR}=\mathrm{mi}$ crowave heating scheme, $\Gamma_{n}$ average neutron flux to the wall, $\Gamma_{h}$ average heat flux to the wall.

There are disadvantages of the stellarator concept but also clear advantages. The high aspect ratio $(R / a>10)$ relaxes numerous technical constraints, e.g., heat load and neutron flux on the inner wall elements. There is no need for strong current drive and ECR-heating is based on the $\mathrm{O} 1$-scheme with already qualified $140 \mathrm{GHz}$ gyrotrons. The stellarator is expected to be more stable (less free energy due to low toroidal plasma currents) and is inherently steady-state capable. There is, however, a number of physics issues that must be carefully addressed by Wendelstein 7-X as described above, notably density profile control and impurity transport control. From the technology point of view, a stellarator fusion power plant has to face challenges but again has also advantages: The magnetic field coils are basically $3 d$-shaped ITER toroidal field coils $\left(\mathrm{Nb}_{3} \mathrm{Sn} 12 \mathrm{~m} \times 7.5 \mathrm{~m}\right)$. This means the coil manufacturing technology will be mostly qualified after the manufacturing of the ITER coils. We note that the $3 d$-shape of the Wendelstein 7-X coils itself was not the major source of problems during manufacturing, testing and assembly. A certain challenge is clearly the divertor geometry, which has to follow a helical path. Also the shape of the wall elements is given by the magnetic field topology and is consequently not perfectly symmetric. This stellarator fusion power plant study provides enough space for blanket elements between plasma surface and coil casing $(1.3 \mathrm{~m})$ and includes sufficiently large vertical ports for maintenance access $(4.3 \mathrm{~m} \times 2.2 \mathrm{~m})$. Maintenance is a critical issue that needs to be addressed and further details can be found in Ref. [16].

\section{Summary}

The construction of Wendelstein 7-X has remained for the last five years on schedule. The last three major work packages are challenging and contain some risks but timely completion is still expected. There is a number of lessons learned from the construction of Wendelstein 7-X which might be useful for the construction of other large research devices. The physics program is under development and is dedicated to the establishment of reactor relevant high-performance steady-state plasma scenarios. The research is focused to address the critical physics issues of a stellarator fusion power plant.

\section{References}

[1] J. Nührenberg and R. Zille, Phys. Lett. A 114 (1986) 129.

[2] V. Bykov et al., Fusion Eng. Des. 86 (2011) 645.

[3] C. Beidler et al., Fusion Technology 17 (1990) 148.

[4] G. Grieger et al., Phys. Fluids B 4 (1992) 2081.

[5] L. Wegener et al., Fusion Eng. Des. 84 (2009) 106.

[6] T. Bräuer et al., IEEE Trans. Plasma Sci. 40 (2012) 577.

[7] Y. Feng et al., Plasma Phys. Controlled Fusion 53 (2011) 024009 .

[8] H. Maaßberg et al., Plasma Phys. Controlled Fusion 41 (1999) 1135.

[9] K. McCormick et al., Phys. Rev. Lett. 89 (2002) 015001.

[10] M. Hirsch et al., Plasma Phys. Controlled Fusion 50 (2008) 053001.

[11] H. P. Laqua et al., Phys. Rev. Lett. 90 (2003) 075003.

[12] Y. Turkin et al., Fusion Sci. Technol. 50 (2006) 387.

[13] J. M. Cranik et al., Phys. Rev. Lett. 98 (2007) 085002.

[14] H. Yamada and S. Sakakibara (Eds.), Fusion Sci. Technol. 58 (2010) (special issue).

[15] Y. I. Kolesnichenko et al., Plasma Phys. Control. Fusion 53 (2011) 024007.

[16] F. Schauer et al., Fusion Eng. Des. (this conference) (2013). 